\title{
RECOPILACIÓN DE INFORMACIÓN VINCULADA A TEMPORALES DE VIENTO, PRECIPITACIONES TORRENCIALES E INUNDACIONES EN LA CIUDAD DE PALMA DE MALLORCA ENTRE LOS AÑOS 2000 Y 2015
}

\author{
José Ma TORRENS CALLEJA, Joan ROSSELLÓ GELI \\ y Miquel GRIMALT GELABERT \\ Grupo de Investigación de Climatología, Hidrología, Riesgos Naturales y Territorio. \\ Universidad de las Islas Baleares. \\ jm.torrens@uib.es, joan.rossellogeli@uib.es, miquel.grimalt@uib.es
}

\section{RESUMEN}

El artículo analiza los sucesos referentes a inundaciones, lluvias torrenciales y vendavales que afectan a la zona de estudio, la ciudad de Palma de Mallorca, en el período comprendido entre los años 2000 y 2015. La metodología utilizada se estructura a partir de la recopilación de noticias aparecidas en la prensa local, y el análisis de datos provenientes de series climáticas. Los resultados obtenidos suponen enlazar la perspectiva de la realidad climática, objeto del estudio científico, y la percepción de la sociedad, a partir de los datos de los medios de comunicación. Se concluye que existe una distorsión entre los valores de los registros meteorológicos y el impacto que dichos fenómenos provocan en la población, reflejados en la prensa. ciones.

Palabras clave: Clima, Prensa, Registros, Precipitaciones, Vendavales, Inunda-

\begin{abstract}
The paper studies the events related to floods, extreme rainfall events and gales that affect the city of Palma de Mallorca between 2000 and 2015. The methodology of the research is based in the use of official climatological data and the damaging hydrometeorological events (DHE) news that appear in the local newspapers. The results link the climatological real data and the societal perception, reflected in the press information. The main conclusion is that a distortion exists between the values measured in the meteorological observations and the impact of DHE within the society as the newspapers reflect.
\end{abstract}

Key words: Climate, Press, Records, Rainfall events, Gales, Floods.

\section{INTRODUCCIÓN}

La ciudad de Palma de Mallorca se ha visto afectada desde inicios del siglo XXI por un conjunto de eventos meteorológicos que pueden considerarse como perjudiciales y son conocidos como DHE (Damaging Hydrometeorological Events) en la literatura científica (Caloiero y Petrucci, 2014; Llasat et al, 2009; Petrucci et al, 2009; UNISDR, 2007). Estos eventos son vendavales y precipitaciones torrenciales que ori- 
ginan daños, directos en el primer caso, y que provocan episodios de inundaciones en el segundo, además de provocar también impactos directos sobre la ciudad y su población. Como fuente para este tipo de investigación, y provocado por la habitual falta de estudios e informes de tipo oficial, se ha venido utilizando la prensa como recurso para la recogida de información sobre los impactos ocasionados por estos eventos extraordinarios, tanto en España (Duce Díaz, 2002; García y Martín, 2000; Pérez García, 2001; Ruíz, 1998) como en otros países europeos (Bayliss y Reed, 2001; Payrastre et al, 2003; Petrucci y Polemio, 2003). La prensa es una fuente accesible, bien en formato papel bien en formato digital, y contiene una información sistematizada (Hernández Varela et al, 2003). Aun así, los datos obtenidos deben ser revisados de forma rigurosa para evitar la recolección de inexactitudes que puedan inducir a errores. En el artículo presentado se procede a una recopilación de las noticias que, sobre eventos extraordinarios, se han publicado en la prensa local de Palma, los diarios Última Hora y Diario de Mallorca. El uso de los medios locales permite presuponer que las noticias tratadas presentan un grado de concreción elevado, al contrario que sucede con medios nacionales en los que el detalle sobre los eventos suele ser sensiblemente inferior (Bayliss y Reed, 2001). Con la información obtenida, se pueden visualizar los episodios estudiados con su interpretación por la prensa local, y comparar su tratamiento con la realidad de los datos meteorológicos obtenidos de las series de observaciones de la AEMET en su estación de Porto Pí en el periodo 2000-2015.

\section{ZONA DE ESTUDIO}

La ciudad de Palma de Mallorca está situada al sur de la isla, en el Occidente del Mar Mediterráneo (Figura 1). Tiene una población de 400.578 habitantes (Ajuntament de Palma, 2015) y es la capital de la isla así como de la comunidad autónoma. Sus orígenes se remontan al año 123 a.C., cuando fue fundada por los romanos. Las sucesivas etapas históricas han dejado su huella en la ciudad, en forma de monumentos y edificios, hasta la actualidad, cuando se ha convertido en una urbe turística, que destaca por su crecimiento urbanístico, iniciado en los años 60 del siglo pasado y que se ha mantenido hasta los inicios de este siglo. Geológicamente hablando, Palma se sitúa sobre una llanura sedimentaria que está cerrada por zonas más elevadas como la sierra de na Burguesa al Oeste o la Costa de Xorrigo al Este. El clima de la zona se corresponde a la clasificación Csa, clima mediterráneo (Koppen, 1948), caracterizado por inviernos suaves y veranos secos. La temperatura media anual es de $17^{\circ} \mathrm{C}$, siendo enero el mes más frio y agosto el más cálido. A nivel de precipitaciones, la ciudad presenta lluvias estacionales, con predominio de las mismas en otoño cuando se alcanzan los máximos registros. Estas lluvias presentan un carácter torrencial y una importante irregularidad (Grimalt, 1989; Sumner et al, 1995). La estación meteorológica utilizada en el estudio es la de Porto Pí (B228), localizada en la zona portuaria de la ciudad, con las coordenadas $39^{\circ} 33^{\prime} 12^{\prime \prime} \mathrm{N}-2^{\circ} 37^{\prime} 31^{\prime \prime}$ E y una altitud de 3 m.s.m. Los datos más destacados de dicha estación aparecen en la Tabla 1.

La ciudad de Palma no es ajena a episodios de precipitación intensa que van acompañados de inundaciones. El espacio urbano se localiza sobre una llanura de inundación por la que circulan varios torrentes, cauces de circulación ocasional, que se han visto afectados por la acción antrópica, en forma de desvíos, canalizaciones, estrechamiento de lechos, etc. Todo ello supone que, desde el siglo pasado, la mayoría 
de eventos se puedan clasificar como inundaciones de carácter local y anegamientos de tierras (Grimalt y Rosselló, 2011). El desarrollo urbanístico en la zona costera ayuda también al incremento de sucesos que se pueden relacionar además con otros factores como la topografía de las zonas afectadas, la falta de adecuación del alcantarillado y por las intensidades de precipitación causantes de los hechos. Los problemas ocasionados por las aguas son básicamente la inundación de plantas bajas y subterráneos así como problemas de tránsito por el corte de calles y pasos inferiores (Grimalt, 1992).

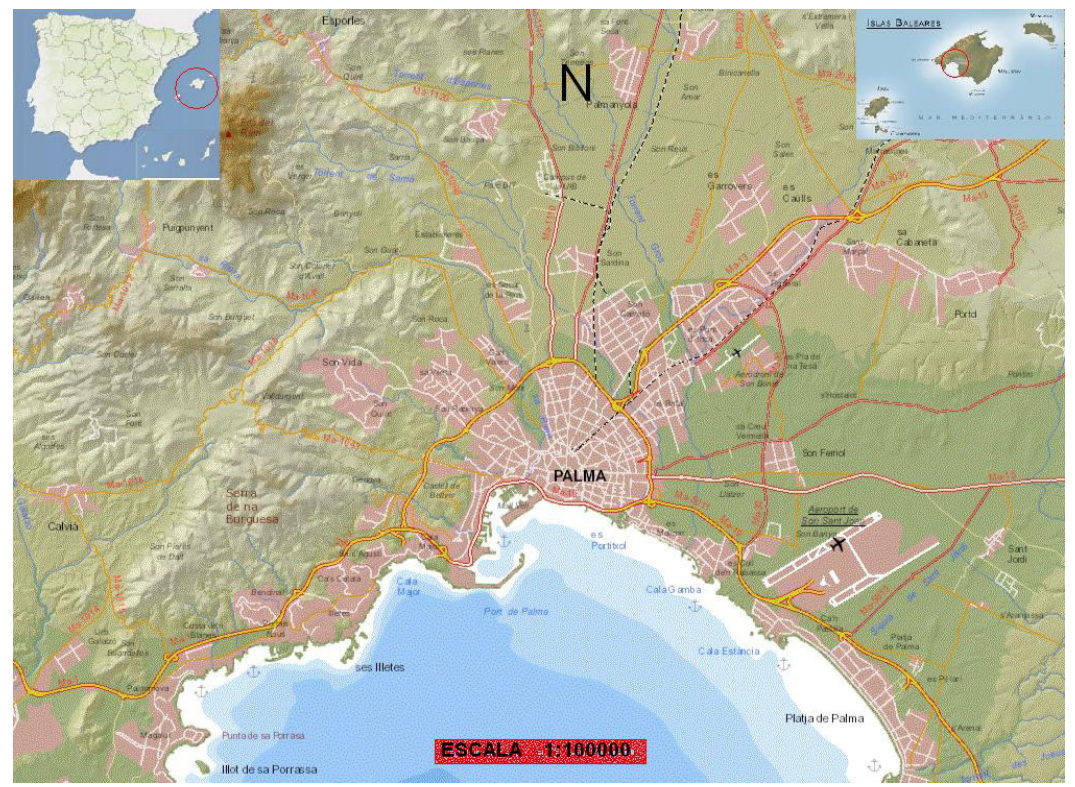

Figura 1. Palma de Mallorca y su localización en la isla. Fuente: elaboración propia a partir de IDEIB.

\begin{tabular}{|c|c|}
\hline Temperatura media & $18,2^{\circ} \mathrm{C}$ \\
\hline Precipitación media & $449 \mathrm{~mm}$ \\
\hline Humedad relativa & $71 \%$ \\
\hline Horas de sol anuales & 2779 \\
\hline Días lluviosos anuales & 53,1 \\
\hline
\end{tabular}

Tabla 1. Variables climáticas estación B228 (1980-2010). Fuente: AEMET.

\section{MÉTODOS}

La metodología utilizada en la realización del artículo se estructura en diferentes etapas. En primer lugar, se ha desarrollado un análisis de los umbrales que permitan calificar a la precipitación y la fuerza del viento. El umbral de precipitación intensa que causa perjuicios en la ciudad de Palma se ha calculado en $30 \mathrm{~mm}$ (Grimalt, 1992) mientras que la intensidad del viento se basa en la escala de Beaufort (1805) conside- 
rando a partir de la categoría 9, temporal fuerte, como las que provocan perjuicios en zonas terrestres (Tabla 2). En segundo lugar se han buscado las fechas con registros diarios que presenten valores por encima de los umbrales anteriormente considerados para el periodo 2000-2015. A partir de estas fechas se ha procedido a una sistemática recopilación de las noticias que, sobre hechos ligados a fenómenos climáticos adversos, han aparecido en la prensa local de Palma. Se han considerado datos como lugares afectados, avisos a los servicios de emergencias, actuaciones de Bomberos y fuerzas de seguridad, daños ocasionados en bienes públicos y privados, etc. Finalmente, se procede a comparar los datos extraídos de la serie climatológica de la estación de referencia (Porto Pí) con los resultados de las búsquedas en la prensa para observar si se corresponde la percepción de la población estudiada con los valores registrados oficialmente.

\begin{tabular}{|c|c|c|c|}
\hline Fuerza & $\mathbf{K m} / \mathbf{h}$ & Clase & Efectos \\
\hline 9 & 75 a 88 & Temporal fuerte & Daños en árboles, imposible andar contra el viento \\
\hline 10 & 89 a 102 & Temporal duro & Árboles arrancados, daños en estructura de construcciones \\
\hline 11 & 103 a 117 & $\begin{array}{c}\text { Temporal muy } \\
\text { duro (borrasca) }\end{array}$ & $\begin{array}{c}\text { Destrucción en todas partes, lluvias muy intensas, } \\
\text { inundaciones }\end{array}$ \\
\hline 12 & Más de 118 & $\begin{array}{c}\text { Temporal } \\
\text { huracanado }\end{array}$ & $\begin{array}{c}\text { Voladura de autos, árboles, casas, techos y personas. } \\
\text { Puede generar un huracán }\end{array}$ \\
\hline
\end{tabular}

Tabla 2. Escala de Beaufort (1805). Fuente: Simón (1997).

\section{REGISTROS CLIMÁTICOS DE VIENTO Y PRECIPITACIÓN}

\subsection{Registros de altas velocidades de viento.}

En el apartado de vendavales que causan perjuicios en la ciudad de Palma, se ha establecido el criterio del umbral a partir de la categoría número 9, temporal fuerte. Los días con valores superiores a este umbral aparecen en la tabla 3.

\begin{tabular}{|c|c|c|c|c|c|c|c|c|c|c|c|c|c|c|}
\hline DIA & MES & AÑO & KM/H & ESC & DIA & MES & AÑO & KM/H & ESC & DIA & MES & AÑO & KM/H & ESC \\
\hline 16 & 2 & 2000 & 101,2 & 10 & 30 & 12 & 2000 & 83,9 & 9,0 & 7 & 9 & 2005 & 81,0 & 9 \\
\hline 3 & 4 & 2014 & 99,0 & 10 & 10 & 11 & 2001 & 83,9 & 9,0 & 8 & 1 & 2010 & 79,9 & 9 \\
\hline 30 & 3 & 2015 & 96,0 & 10 & 15 & 11 & 2001 & 83,2 & 9,0 & 4 & 3 & 2013 & 79,0 & 9 \\
\hline 3 & 3 & 2014 & 93,0 & 10 & 31 & 1 & 2003 & 83,2 & 9,0 & 1 & 2 & 2003 & 78,1 & 9 \\
\hline 5 & 9 & 2000 & 91,1 & 10 & 8 & 12 & 2014 & 83,0 & 9 & 23 & 3 & 2014 & 78,0 & 9 \\
\hline 29 & 10 & 2013 & 91,0 & 10 & 1 & 3 & 2000 & 82,1 & 9 & 29 & 10 & 2007 & 77,0 & 9 \\
\hline 4 & 9 & 2010 & 90,0 & 10 & 1 & 1 & 2010 & 82,1 & 9 & 26 & 12 & 2013 & 77,0 & 9 \\
\hline 13 & 2 & 2005 & 87,8 & 9 & 29 & 2 & 2014 & 82,0 & 9 & 30 & 1 & 2015 & 77,0 & 9 \\
\hline 28 & 2 & 2014 & 87,0 & 9 & 23 & 2 & 2015 & 82,0 & 9 & 29 & 3 & 2014 & 76,0 & 9 \\
\hline 18 & 1 & 2005 & 86,0 & 9 & 21 & 11 & 2015 & 82,0 & 9 & 30 & 11 & 2001 & 76,0 & 9 \\
\hline 4 & 3 & 2014 & 84,0 & 9 & 18 & 5 & 2001 & 81,0 & 9 & 13 & 11 & 2004 & 76,0 & 9 \\
\hline 24 & 2 & 2015 & 84,0 & 9 & 14 & 2 & 2005 & 81,0 & 9 & 2 & 3 & 2015 & 75,0 & 9 \\
\hline
\end{tabular}

Tabla 3. Registros de velocidades de rachas de viento 2000-2015. Fuente: AEMET. 
Analizada mensualmente la tabla 3, el porcentaje de la frecuencia de altas velocidades de viento tiene la siguiente correspondencia: enero $(13,9 \%)$, febrero $(22,2 \%)$, marzo $(22,2 \%)$, abril $(2,8 \%)$, mayo $(2,8 \%)$, septiembre $(8,3 \%)$, octubre $(5,6 \%)$, noviembre $(13,9 \%)$ y diciembre $(8,3 \%)$. Respecto al reparto anual, se distribuye a continuación: 2000 (11,1\%), 2001 (11,1\%), 2003 (5,6\%), 2004 (2,8\%), 2005 (11,1\%), 2006 (0\%), $2007(2,8 \%), 2010(8,3 \%), 2013(8,3 \%), 2014(22,2 \%)$ y $2015(16,7 \%)$. Las rachas de mayor velocidad del viento se localizan, la primera, el día 16/02/2000, $(101,2 \mathrm{~km} / \mathrm{h})$, la segunda el día 03/04/2014, (99,0 km/h), la tercera el día 30/03/2015, (96,0 km/h).

\subsection{Registro de precipitaciones superiores a $30 \mathrm{~mm} / 24$ horas.}

A partir del umbral de los $30 \mathrm{~mm}$ de lluvia, recogidos en 24 horas, se procede al registro de los días en que se ha superado dicha cantidad entre los años 2000 y 2015 (Tabla 4). Estas lluvias, de carácter torrencial, suelen ocasionar perjuicios, en forma de inundaciones, en el espacio urbano de Palma.

\begin{tabular}{|c|c|c|c|c|c|c|c|c|c|c|c|}
\hline DIA & MES & AÑO & MM & DIA & MES & AÑ & MM & DIA & MES & AÑO & MM \\
\hline 4 & 9 & 2015 & 124,3 & 28 & 10 & 2008 & 43,4 & 25 & 9 & 2007 & 33,3 \\
\hline 3 & 5 & 2010 & 112,5 & 29 & 10 & 2013 & 43,4 & 1 & 12 & 2003 & 33,1 \\
\hline 9 & 5 & 2008 & 74,7 & 17 & 10 & 2007 & 43,0 & 30 & 9 & 2015 & 33,0 \\
\hline 24 & 8 & 2002 & 64,0 & 15 & 10 & 2003 & 42,3 & 17 & 9 & 2009 & 32,9 \\
\hline 21 & 10 & 2008 & 60,0 & 23 & 9 & 2010 & 42,3 & 4 & 11 & 2011 & 32,8 \\
\hline 13 & 7 & 2002 & 57,5 & 7 & 3 & 2010 & 42,1 & 28 & 3 & 2007 & 32,5 \\
\hline 19 & 11 & 2013 & 53,3 & 6 & 9 & 2001 & 41,6 & 26 & 8 & 2013 & 32,4 \\
\hline 7 & 6 & 2014 & 52,2 & 24 & 2 & 2006 & 39,9 & 12 & 9 & 2006 & 31,4 \\
\hline 4 & 1 & 2008 & 50,0 & 15 & 12 & 2008 & 39,2 & 15 & 8 & 2014 & 31,4 \\
\hline 8 & 1 & 2010 & 47,4 & 24 & 2 & 2001 & 37,9 & 27 & 4 & 2007 & 31,3 \\
\hline 7 & 11 & 2011 & 46,9 & 20 & 8 & 2005 & 37,9 & 18 & 5 & 2008 & 30,9 \\
\hline 11 & 11 & 2014 & 46,8 & 6 & 11 & 2011 & 35,9 & 31 & 5 & 2011 & 30,8 \\
\hline 15 & 11 & 2012 & 46,0 & 22 & 9 & 2007 & 35,1 & 18 & 11 & 2013 & 30,6 \\
\hline 9 & 8 & 2002 & 45,3 & 15 & 9 & 2009 & 35,1 & & & & \\
\hline
\end{tabular}

Tabla 4. Fechas de las precipitaciones mayores de 30 mm/día. Fuente: Aemet

De los meses de la tabla representada el porcentaje de la frecuencia de altas precipitaciones tiene la siguiente correspondencia: enero $(4,9 \%)$, febrero $(4,9 \%)$, marzo $(4,9 \%)$, abril $(2,4 \%)$, mayo $(9,8 \%)$, junio $(2,4 \%)$, julio $(2,4 \%)$, agosto $(12,2 \%)$, septiembre $(22,0 \%)$, octubre $(12,2 \%)$, noviembre $(17,1 \%)$ y diciembre $(4,9 \%)$. Respecto a los años se atribuye a continuación: 2001 (4,9\%), 2002 (7,3\%), $2003(4,9 \%), 2005$ (2,4\%), 2006 (4,9\%), 2007 (12,2\%), 2008 (14,6\%), 2009 (4,9\%), 2010 (9,8\%), 2011 (9,8\%), $2012(2,4 \%), 2013(9,8 \%), 2014(7,3 \%)$ y $2015(4,9 \%)$. De los registros de la tabla, el 48,8\% están entre $30-40 \mathrm{~mm}$, el $29,3 \%$ entre $40-50 \mathrm{~mm}$, el 9,8\% entre $50-60$ $\mathrm{mm}$, el 4,9\% entre 60-70 mm, el 2,4\% entre 70-80 $\mathrm{mm}$ y el 4,9\% de más de $100 \mathrm{~mm}$. La mayor precipitación diaria es el día 04/09/2015 (124,3 $\mathrm{mm})$, la segunda mayor es $03 / 05 / 2010(112,5 \mathrm{~mm})$ y la tercera mayor es $09 / 05 / 2008(74,7 \mathrm{~mm})$. 


\section{EPISODIOS DE INUNDACIÓN EN EL PERIODO 2000-2015}

A partir del análisis de la prensa local de Palma, se han podido identificar 27 episodios que pueden considerarse como DHE entre los años 2000 y 2015. Estos casos se han clasificado según la fecha de publicación de la noticia y se acompaña la información recogida en la prensa con los datos oficiales de la estación B228 de la AEMET (Tabla 5), referidos a la precipitación recogida en 24 horas y la intensidad en $\mathrm{km} / \mathrm{h}$ de la ráfaga máxima de viento y su fuerza según la escala de Beaufort. De la información de los periódicos se obtienen las zonas afectadas, que se clasifican en 3 grupos (Figura 2): (1) casco antiguo (que corresponde a la zona centro de Palma), (2) ensanche (que está formado por las barriadas que se construyeron a lo largo del siglo XX envolviendo la zona central) y (3) periferia (donde se incluyen los polígonos industriales y de servicios, el aeropuerto y la zona turística del Arenal). Así mismo, los datos recabados en la prensa, permiten dividir los daños ocasionados en dos grandes grupos, que a su vez se subdividen en varias categorías. Por un lado encontramos los daños causados por el viento (Tabla 6) y, por el otro, los ocasionados por las inundaciones (Tabla 7).

\begin{tabular}{|c|c|}
\hline Categoría de daños por viento & Efectos \\
\hline A1 & Caída de tejas, elementos de fachadas \\
\hline A2 & Caída de árboles \\
\hline A3 & Caída de mobiliario urbano (farolas, señales) \\
\hline A4 & Daños a vehículos y propiedades privadas \\
\hline
\end{tabular}

Tabla 6. Daños causados por efectos del viento.

\begin{tabular}{|c|c|}
\hline Categoría de daños por inundaciones & Efectos \\
\hline B1 & Anegamiento calles, caminos y carreteras \\
\hline B2 & Inundación subterráneos y bajos \\
\hline B3 & Cortes transportes públicos y privados \\
\hline B4 & Daños a vehículos y propiedades privadas \\
\hline
\end{tabular}

Tabla 7. Daños causados por las inundaciones.

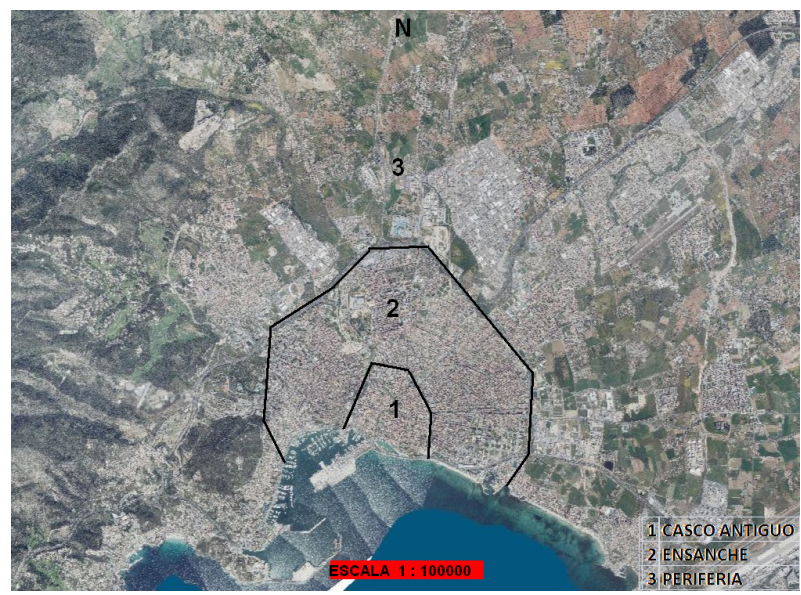

Figura 2. Distribución de las zonas afectadas. Fuente: elaboración propia a partir de IDEIB. 


\begin{tabular}{|c|c|c|c|c|}
\hline Fecha & $\begin{array}{c}\text { Precip. } \\
(\mathrm{mm} / \mathbf{2 4 h})\end{array}$ & $\begin{array}{c}\text { Ráfaga } \\
\text { (Fuerza) }\end{array}$ & $\begin{array}{c}\text { Zonas } \\
\text { afectadas }\end{array}$ & Daños \\
\hline $06 / 09 / 2001$ & 41,6 & $45,0(\mathrm{~F} 6)$ & 3 & $\mathrm{~A} 1, \mathrm{~B} 1, \mathrm{~B} 2$ \\
\hline $13 / 07 / 2002$ & 57,5 & $36,0(\mathrm{~F} 5)$ & $1,2,3$ & $\mathrm{~B} 1, \mathrm{~B} 2, \mathrm{~B} 3, \mathrm{~B} 4$ \\
\hline $20 / 08 / 2007$ & 25,4 & 29,2 (F5) & 2,3 & B3 \\
\hline $24 / 09 / 2007$ & 27,1 & $20,9(\mathrm{~F} 4)$ & 2 & B3 \\
\hline $04 / 10 / 2007$ & 22,3 & $109,1(\mathrm{~F} 11)$ & 2,3 & $\mathrm{~A} 1, \mathrm{~A} 2, \mathrm{~A} 3, \mathrm{~A} 4, \mathrm{~B} 1, \mathrm{~B} 2, \mathrm{~B} 3, \mathrm{~B} 4$ \\
\hline $12 / 10 / 2007$ & 43,0 & $61,9(\mathrm{~F} 8)$ & 3 & $\mathrm{~A} 2, \mathrm{~B} 1$ \\
\hline $17 / 10 / 2007$ & 22,3 & 34,9 (F5) & $1,2,3$ & $\mathrm{~B} 1, \mathrm{~B} 2, \mathrm{~B} 3, \mathrm{~B} 4$ \\
\hline 09/05/2008 & 74,7 & $33,8(\mathrm{~F} 5)$ & 1,2 & B1, B2, B3 \\
\hline $18 / 05 / 2008$ & 30.9 & $25,9(\mathrm{~F} 4)$ & 2 & B2 \\
\hline $01 / 09 / 2008$ & 11,1 & $28,1(\mathrm{~F} 5)$ & 2 & $\mathrm{~B} 2$ \\
\hline $22 / 09 / 2008$ & 12,4 & $11,9(\mathrm{~F} 2)$ & 2,3 & $\mathrm{~B} 1, \mathrm{~B} 2, \mathrm{~B} 3$ \\
\hline $12 / 10 / 2008$ & 4,9 & $46,1(\mathrm{~F} 6)$ & 2 & B2 \\
\hline $15 / 12 / 2008$ & 39,2 & $37,1(\mathrm{~F} 5)$ & 2 & $\mathrm{~B} 2, \mathrm{~B} 3$ \\
\hline $22 / 09 / 2009$ & 14,0 & $33,1(\mathrm{~F} 5)$ & 2,3 & $\mathrm{~A} 2, \mathrm{~B} 1, \mathrm{~B} 2, \mathrm{~B} 3$ \\
\hline $28 / 09 / 2009$ & 23,4 & $38,9(\mathrm{~F} 6)$ & $1,2,3$ & $\mathrm{~A} 1, \mathrm{~A} 2, \mathrm{~B} 1, \mathrm{~B} 2, \mathrm{~B} 3, \mathrm{~B} 4$ \\
\hline $08 / 01 / 2010$ & 47,4 & 79,9 (F9) & 1,2 & $\mathrm{~A} 1, \mathrm{~A} 2, \mathrm{~A} 3, \mathrm{~A} 4, \mathrm{~B} 1, \mathrm{~B} 3$ \\
\hline $03 / 05 / 2010$ & 112,5 & 54,0 (F7) & $1,2,3$ & B1, B2, B3, B4 \\
\hline $31 / 05 / 2011$ & 30,8 & $36,0(\mathrm{~F} 5)$ & $1,2,3$ & $\mathrm{~A} 1, \mathrm{~A} 2, \mathrm{~B} 1, \mathrm{~B} 2$ \\
\hline $04 / 11 / 2011$ & 15,0 & $100,0(\mathrm{~F} 10)$ & 1,2 & $\mathrm{~B} 1, \mathrm{~B} 2, \mathrm{~A} 1, \mathrm{~A} 2$ \\
\hline $14 / 11 / 2011$ & 80,0 & $30,0(\mathrm{~F} 5)$ & 1,2 & $\mathrm{~A} 1, \mathrm{~A} 2, \mathrm{~A} 3$ \\
\hline $26 / 08 / 2013$ & 32,4 & $26,0(\mathrm{~F} 4)$ & $1,2,3$ & $\mathrm{~A} 2, \mathrm{~B} 1, \mathrm{~B} 2, \mathrm{~B} 3$ \\
\hline $29 / 10 / 2013$ & 43,4 & $91,0(\mathrm{~F} 10)$ & 1,2 & $\mathrm{~A} 1, \mathrm{~A} 2, \mathrm{~A} 4, \mathrm{~B} 1, \mathrm{~B} 2, \mathrm{~B} 3$ \\
\hline $18 / 11 / 2013$ & 30,6 & $31,0(\mathrm{~F} 5)$ & 2 & $\mathrm{~A} 1, \mathrm{~A} 2$ \\
\hline $19 / 11 / 2013$ & 53,5 & $45,0(\mathrm{~F} 6)$ & 1,2 & $\mathrm{~A} 1, \mathrm{~A} 2$ \\
\hline $17 / 06 / 2014$ & 69,0 & $40,0(\mathrm{~F} 6)$ & $1,2,3$ & $\mathrm{~B} 1, \mathrm{~B} 2, \mathrm{~B} 3, \mathrm{~B} 4$ \\
\hline $04 / 09 / 2015$ & 124,3 & $60,0(\mathrm{~F} 7)$ & $1,2,3$ & $\mathrm{~A} 1, \mathrm{~A} 2, \mathrm{~A} 3, \mathrm{~A} 4, \mathrm{~B} 1, \mathrm{~B} 2, \mathrm{~B} 3, \mathrm{~B} 4$ \\
\hline $30 / 09 / 2015$ & 33,0 & $31,0(\mathrm{~F} 5)$ & 3 & $\mathrm{~B} 1, \mathrm{~B} 2$ \\
\hline
\end{tabular}

Tabla 5. Episodios con registros de daños en Palma. Fuente: elaboración propia a partir de datos Aemet, Diario de Mallorca y Última Hora.

\section{CONCLUSIÓN}

Se aprecia como la mayoría de episodios con daños afectan al espacio geográfico formado por la zona centro y el ensanche de Palma, mientras que aparece un menor número de casos en la periferia. Por lo que se refiere a los tipos de daños, destacan los provocados por los anegamientos, que son mayoritarios, sobre todo las inundacio- 
nes de bajos y subterráneos así como los cortes de calles y las dificultades para los transportes tanto públicos como privados. En relación al viento, sus efectos se dejan sentir también, pero son cuantificados por la prensa en pocas ocasiones. Por lo que se refiere a las cantidades de precipitación que originan impactos por inundación, se aprecia en los registros que son suficientes cantidades por encima de los $20 \mathrm{~mm} / 24$ horas para originar problemas en la zona urbana. Incluso se dan en 4 ocasiones impactos con cantidades entre 10 y $20 \mathrm{~mm}$. En cambio, los problemas originados por el viento destacan cuando las velocidades máximas se sitúan en valores de Fuerza 5 y superiores, alcanzando su máxima expresión, según los datos recogidos por la prensa, con rachas de fuerza 9, 10 e, incluso, fuerza 11. Los meses más representados con las rachas más intensas de viento superiores a fuerza 9, son el mes de febrero y marzo. En la representación de las precipitaciones de más de $30 \mathrm{~mm}$ diarias, el mes con altas precipitaciones es septiembre, seguido por orden de noviembre, octubre y agosto. En las inundaciones históricas los meses según los eventos de inundaciones son: octubre, septiembre y agosto. Las inundaciones en la ciudad de Palma, no dependen directamente de la precipitación acumulada en la ciudad en 24 horas. Más bien es un factor condicionante. Las inundaciones se producen por cuatro factores: la intensidad horaria de la precipitación, la zona de localización de la precipitación, la topografía del relieve en zonas de inundables de torrentes y la intervención humana en la urbanización del territorio. Las fuentes de información de la prensa local, permiten conocer los eventos de inundaciones en lugares que, por falta de cobertura, no están cubiertos por estaciones meteorológicas dentro de la ciudad. De esta manera se permite conocer la percepción objetiva de la realidad de los efectos de las inundaciones en el municipio de Palma.

\section{AGRADECIMIENTOS}

Agradecimientos a la Universitat de les Illes Balears, a la prensa local de Palma, a la Delegación territorial de la Aemet y a la biblioteca municipal de Palma Can Sales.

Trabajo desarrollado en el marco del proyecto HyMeX.

\section{REFERENCIAS}

Ajuntament de Palma (2015): Dades de població. Ajuntament de Palma.

Bayliss, A.C., y Reed, D.W. (2001): The use of historical data in flood frequency stimation. Report to MAFF. Centre for Ecology and Hydrology. Wallingford. 92 pp.

Caloiero, T., y Petrucci, O. (2014): The impact of Damaging Hydrogeological Events on urbanized sectors: the case of $19^{\text {th }}$ November 2013 in Catanzaro (Italy). $3^{\text {rd }}$ IAHR Europe Congress. Book of Proceedings. Oporto.

Duce, E. (2002): El medio natural como problema social: los aspectos hídricos en el diario. La Vanguardia (1970-1900)

Guijarro, J., Grimalt, M., Laita, M., y Alonso, S. (2004):. L'aigua i el clima. Publicaciones de la A.E.C. serie A n ${ }^{\circ}$ 3. Mallorca, pp. 407-416.

García, E., y Martín, A. (2000): Riesgos climáticos en Galícia: una aproximación a través de la prensa (1983-1997). Ería, 53, pp. 259-270. 
Grimalt, M. (1989): Repartiment de les precipitacions màximes a Mallorca. Treballs de Geografia, 41, pp. 7-18.

Grimalt, M. (1992): Geografia del risc a Mallorca. Les inundacions. Institut d'Estudis Baleàrics. Palma de Mallorca. 360 pp.

Grimalt, M., y Rosselló, J. (2011): Anàlisi històrica de les inundacions a les Illes Balears. Conselleria d'Agricultura, Medi Ambient i Territori. Govern de les Illes Balears. 442 pp+atlas.

Hernández V., Lozano, MA., y Soleto, C. (2003): Estudio de los acontecimientos meteorológicos extraordinarios en la comunidad autónoma del País Vasco (18701954) a través de la prensa. Investigaciones Geográficas, nº 30, pp. 165-180.

Köppen, W. (1948): Climatología, con un estudio de los climas de la tierra. Fondo de Cultura Económica. México.

Llasat, M.C., Llasat-Botija, M., Barnolas, M., López, L., y Altava-Ortiz, V. (2009): An analysis of the evolution of hydrometeorological extremes in newspapers: the case of Catalonia, 1982-2006. NHESS, 9, 1201-1212.

Payrastre, O., Gaume, E., y Andrieu, H. (2003): Use of local and regional newspapers for historical flood investigation in small catchment areas. EGS-AGU Joint Assembly Abstracts. Niza, Francia.

Pérez, A.P. (2001): Avenidas fluviales e inundaciones en el Medio Vinalopó en el tránsito de los siglos XIX y XX. Investigaciones Geográficas, nº 26, pp. 169-181.

Petrucci, O., y Polemio, M. (2003): The use of historical data for the characterization of multiple damaging hydrogeological events. NHESS, 3, 17-30.

Petrucci, O., Polemio, M., y Pasqua, A. (2009): Analysis of Damaging Hydrogeological Events: the case of Calabria region (Southern Italy). Environmental Management, 43 (3), pp. 483-495.

Ruíz, E. (1998): El clima del País Vasco a través de la prensa. Ediciones del Servicio Vasco de Meteorología. Gobierno Vasco. 211 pp.

Simón, J. (1997): Patrones de embarcaciones de recreo. José de Simón Quintana. Cádiz. 574 pp.

Sumner, G., Ramis, C., y Guijarro, JA. (1995): Daily rainfall domains in Mallorca. Theoretical and Applied Climatology. Volume 51, Issue 4, pp. 199-221.

UNISDR (2007): Terminology on DDR. United Nations Office for Disaster Risk Reduction. Acceso online 20-03-2016. Recuperado de: www.unisdr.org/we/ inform/terminology 